\title{
On C-E Translation of Movie Names Based on Skopostheorie
}

\author{
Wei Zhang, Xue Chen, Xinyu Bu
}

School of Foreign Languages, Sanjiang University, Nanjing 210012, China

\begin{abstract}
Movie name, as an indispensable component of the movie as a whole, is used to transmit the ideas of the movies, please audiences with aesthetic value, and enhance the interests of watchers to watch it. Skopos theory highlights the importance of both the translator and the culture and it makes the translator find a suitable mode to translate the names of movies. Movie name translation has become a important part of translation studies. Skopos theory can lead the translator to do a rendering of movie names while he/she knows the objectives of translation.
\end{abstract}

Keywords: Movie Name; Skopostheorie; Translation Method

COPYRIGHT

Copyright (C) 2019 by author(s) and Frontier Scientific Publishing. This work is licensed under the Creative Commons

Attribution-NonCommercial 4.0 International License (CC BY-NC 4.0).

http://creativecommons.org/licenses/

\section{Introduction}

Movie, due to its unique characteristics has become one of the most popular existence of mass media. It reflects not only disparate social sides and different cultures but also shows how to fulfill the psychological needs of the target audience by having them experience the living things of others. To some degree, movies indeed impact the society from all kinds of aspects. The rendering of movie name is not a very simple and easy job, yet in effect a very complex as well as demanding work. By adopting Skopos theory into movie name translation, the translator can get a deep insight into the purposeful basis of movie names by looking into the whole history, cultures and values to do movie name translation in addition to commercial motivations. Furthermore, the previous studies guided by Skopos Theory is not enough. The rendering of movie names is indeed a intentional thing. Due to this, studies on the Chinese-English translation based on Skopos Theory of movie names is a inevitable thing.

\section{Literature Review}

The Skopos theory, or Skopostheorie, was put forth by Vermeer. The suppositions and inference of the theory manifest his thoughts and concept. Skopostheorie is the foundation and basis of the functional grammar translatio theory. In addition to Vermeer, Christiane Nord is another principal and major celebrity, who summarizes the prior studies on Skopostheorie and writes a monograph Rendering as a Purposeful Activity: Functionalist Approaches Explained, which has given a in-depth and methodical examination of the functional grammar theory including Skopostheorie. At the present time, the roles of the theory impact more and more specific points. There have appeared more and more works on Skopostheorie in recent years. The translation cricle has attached more attention to this theory. For one thing, the domestic study on Skopostheorie itself is not so advanced and edged like abroad; for another, the application 
of this theory in domestic circle is not adequate and extensive enough, though quite a few researchers having carried out their studies from different perpectives

\section{Skopostheorie}

\subsection{Definition of Skopostheorie}

Skopostheorie was first introduced by Hans J. Vermeer in 1970s, and the major work on Skopostheorie is his book Groundwork for a General Theory of Translation. Skopos is an extremely crucial concept in Skopos Theory. Generally, "Skopos" refers to the communicative skopos of the translations. Vermeer believes that translation is not only an activity of human, but also an act with purposes. The core concept of Skopos Theory is translation methods and translation methods must be determined by the expected purposes or functions of the translations.

\subsection{Three rules of Skopostheorie}

After detailing the definitions of the basic concepts of the theory, further puts forward the skopos' rule which is viewed as the top-ranking rule in translation. They are Skopos Rule, Coherence Rule and Fidelity Rule. Among the three guiding rules of Skopos Theory, the skopos rule should be regarded as the top-ranking rule for any translation attached much importance. Besides, the loyalty principle put forward by Christiane Nord is also indispensable and can be regarded as a complement to the three rules raised by Vermeer.

\subsubsection{Skopos Rule}

The prime principle in Functionalist Skopos Theory is the "skopos rule". Vermeer considers that human interaction (including translation) is determined by the purpose and translation follows a "purpose". According to Nord, there are three possible kinds of purposes in the field of translation: 1) the translator's general purpose in the translation process; 2) the communicative purpose aimed at by the target text in the target situation; 3 ) the purpose aimed at by a particular translation method or procedure. Skopos rule mainly refers to that the target language should be able to play a role in the culture and context of the target language in the way which the addressees expect. Therefore, this rule is always opposite to the traditional translation rule on the basis of equivalence. According to the Skopos Rule, the translator is able to adopt the methods of translation freely since the translation is all depending on the intended Skopos for which the translation is needed.

\subsubsection{Coherence Rule}

Another principal rule of Skopos Theory is the coherence rule, which means that "a translation should be acceptable in a sense that it is coherent with the receivers' situation". Coherence Rule refers to the translation needs to be consistent with the criterion of intratextual coherence. It means that the translations must be capable of being understood by receivers and readable, and also be meaningful in the communication situations of the target language's culture and its translations. Therefore, the translator should take full account of cultural backgrounds and social environment of target addressees to create the translation making sense for the addressees, that is, the target text should achieve semantic coherence to the largest extent with readability and acceptability to make addressees understand its meaning. Only in this way can information be communicated successfully.

\subsubsection{Fidelity Rule}

Since translation is a new conveyance from the source information to the target information, it shall keep in touch with the source text to some extent. This is "Fidelity Rule". Fidelity Rule means the translator should not only respect the source-text author but also be responsible for the target reader while rendering. It emphasizes on translation conversion and expects to establish a specific relationship between the source language and language---"intertextual coherence" or "fidelity" as well as requires a certain relationship maintained between the source language and target 
language. It also needs to achieve a consistent relationship among the source-text author, translation initiator and the target reader. In Skopostheorie, fidelity rule is considered subordinate to coherence rule, and both are subordinate to the Skopos rule.

\subsection{Relationship among the Three Rules}

The three basic rules of Skopos Theory are designed to manipulate the whole translation process. However, they are not of equal importance concerning their manipulating power. Skopos Rule is the first and the most principal rule of the three rules. Fidelity Rule is subject to coherence rule, but both are subordinated to Skopos rule. Then Coherence Rule is no longer valid. If the skopos of translation asks the target text to represent the content and style of the source text, then Fidelity Rule.

\subsection{Merits of Skopostheorie}

By taking precedence over purpose and function in translation, Skopos Theory brings a new perspective on translation studies. As the great breakthrough of Western translation studies, Skopos Theory has several merits as follows: First, Skopos Theory regards translation as a kind of human act with particular purposes, which is a complex activity that switches all of verbal symbols and non-verbal symbols of one language to another language rather than the simple conversion of the linguistic level. Second, Skopos Theory breaks through traditional translation theories, and gives a way to the long-time perplexed questions, such as literal translation \& free translation, domesticating \& foreignizing and questions about faithfulness and so on. Third, it proposes a Skopos Rule-driven and multicriteria theoretic system. In real life, translation problems that encountered by the translator are various.

A single criterion of translation is unable to solve these problems.

\section{Analysis of the English Movie names from the perspective of Skopostheorie}

The movie name translation is a process of creative efforts with a clear skopos, that is, to call upon the intended audience in the target culture to the movie. Skopos Theory mainly analyzes translation process, translation methods and translation evaluation from the skopos of the target text. It provides a new translation perspective for the translator and puts forward new and flexible translation methods. In the process of translation, the initiator of the target text determines the skopos of the translations. Obviously, Skopos Theory provides a strong theoretical explanation for many phenomena of movie names. The key point of Skopos Theory is not only to achieve the cultural, artistic value of the movie, but also attract the audience to the greatest extent and realize the commercial value of the movie. Skopos Theory analyzes the source text and settles on the translation methods from the skopos of the target text, which is no longer in one-sided pursuit of the mechanical equivalence between the target text and the source text. It also provides a theoretical basis for adopting a variety of alternative means in the course of English movie names' translation. Skopos Theory holds the skopos of translation acts determines the translation methods and methods. The main methods of English movie names' translation guided by Skopos Theory are Transliteration, Literal Translation, Liberal Translation, Complementary Translation and Creative Translation.

\subsection{Transliteration}

Transliteration is to seek phonetic correspondence in terms of the translation of proper names in movie names, especially the names of persons and the places and historical events which are very familiar to the audience or foul of historical and cultural connotations. In other words, transliteration refers to rendering movie names by taking into account the sounds of words instead of their verbal meanings. Nowadays, as people show more and more interest in getting familiar with other country's culture, this method is becoming increasingly paramount. A few examples are given below:Troy《特洛伊》; Titanic《泰坦尼克》; Mulan《花木兰》.

Transliteration enables the target audience to know more about the original history and historical figures. What's 
more, many names are already of great cognitive value and having attracted the audience. If they are translated in other ways, the moviegoers may think that it is just an ordinary movie. Thus they may have no interest in seeing it. Therefore, many movies adapted from famous literary works just follow the transliterated names of the original, such as:Pride and Prejudice《傲慢与偏见》; Jane Eyre《简爱》; Romeo and Juliet《罗密欧与朱丽叶》. This translation not only is in accordance with the content of the movie, but also conveys connotation of the original movie pertinently without constraint of English name.

Transliteration also applies on the movies rewritten on the basis of well-known historic events, or about celebrities, historic figures and well-known places. Because these names of persons, places and events have some cultural and historical significance for people, and this kind of movie name is the best signboard itself. Just as Gandi 《甘地》, Casablamca 《卡萨布兰卡》, Peter Pan《彼得潘》and so on.

\subsection{Literal Translation}

Literal translation, which is word-for-word translation aims to produce a translation which retains the content and form of the original movie name. Literal translation can reproduce both form and content of the original in most cases. Such names can be directly translated into Chinese. We can even say these movie names "need no translation". This kind of names is usually nouns or noun phrases. For example:Brave Heart《勇敢的心》; Star Wars《星球大战》; Rain Man 《雨人》. More and more foreign movie names are translated literally nowadays. Literal translation can not only offer the cultural information, but also enrich the culture of target language. With the increasing cultural communication among the people from different countries people of different culture could understand each other better than before. Under this circumstance, literal translation serves the appellative function of the movie name well. In translation, we should know that there is a vital difference in thinking ways between Westerners and Chinese. Westerners are apt to think in terms of abstraction, that is abstract thinking. The Literal translation is adopted in many cases as a useful technique in rendering movie names.

\subsection{Liberal Translation}

Liberal translation makes quite a lot changes in linguistic properties of the original text. It is difficult to realize the functional equivalence in the movie name translation using liberal translation. Liberal translation is a method that seeks to convey the meaning and the spirit of the original name without sticking to the form mechanically. It can be seen that in movie name translation it is impossible to achieve "faithfulness" at the language level in many cases. Liberal translation is most frequently applied when it is really impossible for the translator to use the methods previously discussed. Sometimes, when the literal translation fails to reach the goal of successful translation, liberal translation is employed. These are typical examples of liberal translation in movie name translation: Alive 《劫后余生》; Ghost《人 鬼情未了》. The strongpoint of this technique is that such combination can transmit the message to the most extent and add to the artistic appeal of the name since literal translation can deliver the literal meaning of the name at surface level, and at the same time liberal translation expresses the implied meaning of the name in depth.

\subsection{Combination of Transliteration and Liberal Translation}

The combination of liberal translation and transliteration as a translation technique can be adopted to achieve the information, expressive and vocative functions of movie. This method means that the name of the movie can be translated by combining phonetic features and meanings. Let's take 《Titanic》 as an example. Titanic is a luxury liner which had been proclaimed unsinkable. The sinking of the Titanic, as a famous historical event, is familiar to the people of English-speaking countries. However, most of the Chinese audience lack the necessary background information of this event. Therefore, in rendering this movie name, the transliteration 《 Titanic 》 cannot give the audience any helpful information. The translation not only shows that audience its referential function but also presents its appellative function by attracting the audience to watch the movie to see what happened to this ship. 
More similar examples are listed as following: The Muse《缪斯女神》; Patton《巴顿将军》.

\subsection{Creative Translation}

Creative translation is a specific method of translation variations. Changes of creative translation should give the audience the enjoyment of wonder on basis of cultural identity or experience. The following are some good examples. If the name Top Gun is translated into “顶部的枪”, there is no doubt it makes no sense to Chinese audience. What does it mean? Top Gun means the United States Navy Fighter Weapons School. Meanwhile, in English top gun means "the best in best". So, literal translation is unacceptable. Moreover, the literal translation“鬼 ”is certainly unacceptable. People would regard it as a thriller at the first sight. Then the translator combines metaphrase with paraphrase. Its Chinese translation is 《人鬼情未了》, which tells us the characters and the theme of the movie. There are some other such masterpieces: “Top Gun”《壮志凌云》, “Leon”《这个杀手不太冷》, “Sleepless in Seattle”《缘分的天空》, “Legend of the Fall” 《燃情岁月》 and so on. In accordance with the content of the movie, the translator makes a creative translation which enables to it attract the audience's attention to its maximum.

\section{Conclusion}

The movie is one of the most enchanting and impactful public mass media products. As an important integral component of the movie, movie name, not just appeals the audiences, but also improves the movies' historical name and status. Rendering movie names is an engrossing, consequentia as well as taxing job. The rendering of movie names becomes an increasing important task movies. Although related topics have sometimes been tackled in some papers, yet they haven't give out a systematic translation theory to guide the C-E translation of movie name. The Skopos in C-E translation is not necessary to realize equivalence which should be one of translator's purposes. Besides, under the guidance of Skopos Theory C-E translation of movie names will be capable of better assisting studies on movie names' translation, showing the attractiveness and glamour of movies, fulfill the artistic and emotional value of movies, and demonstrates stylish commercial worth and use of the movie.

\section{References}

1. Nord, Chriatian. (2001) Translation as a Purposeful Action[M]. Shanghai Foreign Language Education Press, Shanghai.

2. Newmark, P.(1978) Translation as Adaptation and Selection [M]. Heidelberg University, Heidelberg.

3. Nida, E.A \& Taber. C. R. (1993) The theory and practice of translation [M]. Shanghai Foreign Language Education Press, Shanghai. 